\title{
Nothing is Certain Except Taxes and the Other Thing: Searching for Death Anxiety in a Large Online Sample
}

\author{
Sina Storelv \& Bjørn Sætrevik \\ Department of psychosocial science, Faculty of psychology, University of Bergen
}

\begin{abstract}
Philosophical and psychological literature has suggested that death anxiety has a profound impact on people's lives and is a fundamental aspect of what it means to be human. Such claims motivated us to examine how people express their emotions and attitudes toward death in short free-text responses. We explored a qualitative dataset where 803 Americans stated their thoughts about either death or physical pain (toothache). Comparing these, we found that death prompts caused the expression of less negative affect and more positive affect than toothache prompts. The reactions to death were quite diverse and did not appear to be dominated by existential anxiety. We did not see compelling evidence for psychological defense mechanisms causing the underreporting of negative affect toward death. The article serves as a companion for an open dataset, to allow other researchers to explore and reuse it.
\end{abstract}

Keywords: Death anxiety, death thoughts, terror management theory, psychological defense mechanisms, content analysis 
How do people, as conscious and mortal beings, cope with the realization that they one day will die? Philosophers and psychological scholars (such as Becker, 1973; Freud, 1915; Greenberg et al., 1986; Kierkegaard, 1849; and Nietzsche, 1901) have argued that becoming aware of death causes great existential anxiety, often known as "death anxiety". They have argued that the anxiety and distress caused by this death awareness significantly impacts people's motivation and behavior and shapes cultures and society. Such grand claims are not made for other types of distress, which implies that death anxiety is a distinct human experience.

Inspired by such claims, we performed an explorative study of how people describe their emotions toward death compared to their emotions toward a physical pain. Although this study was not designed to evaluate theories about how people relate to death, exploring this dataset may provide insight into people's thoughts about their mortality and inspire future research questions. To our knowledge, there are few comparable datasets publicly available (one exception can be found here: Nilsson \& Sinclair, 2018).

\section{Theoretical approaches to how people relate to death}

Existential philosophers such as Kierkegaard and Nietzsche emphasized how awareness of death impacts people's spirituality (Bonthius, 1948; Nietzsche, 1901). Nietzsche (1901) stated that people strive for spiritual immortality and a higher purpose, and they are drawn to cultural and spiritual sy stems to achieve this. Kierkegaard (1849) argued that what he called a "sickness unto death" is an inherent factor of being human, and that people may relieve this condition by establishing a vital relationship with God (Bonthius, 1948). Without this relationship with God, people are subject to existential dread and despair that comes with being able to contemplate one's mortal existence.

In the influential book The Denial of Death (Becker, 1973, p. xvii) argued that people's death anxiety is a universal, natural and basic fear that "haunts the human animal like nothing else". This existential anxiety will motivate people to pursuit projects that makes them feel a part of something that will last longer than their own lives. Devoting oneself to religion may be such a project, or it could be publishing a book or having children. If they do not have such a project, people are instead drawn to hedonistic pleasures such as drugs or alcohol use, or materialism. In other words, humans seek out certain activities to repress death anxiety, and this anxiety is therefore one of the primal forces of motivation for people's behavior.

Ideas from Becker's work were formalized into the "terror management theory"(TMT). This influential framework in psychology argues that being aware of one's mortality leads to an overwhelming existential anxiety ("terror") that needs to be resolved (Greenberg et al., 1986; Pyszczynski et al., 2015). Resolution of this anxiety can come through two different mechanisms: Either through actions and mindsets that increase one's self-esteem and protect one's worldview, or intra-psychologically through denying or suppressing the thought of death. The TMT has formed the framework for several hundred experiments on the effects of death reminders, often known as mortality salience (MS) effects. This research tradition has attempted to show associations between reminders of one's mortality and increased in-group bias and protection of one's worldview (Burke et al., 2010, 2013). A considerable number of empirical studies have claimed to support MS effects. At the time of writing, the term "mortality salience" returns 15,500 results on Google Scholar. However, there is substantial variation among the studies, without the emergence of a clear consensus on an experiment design that should support a given hypothetical claim. Further, most of the studies claiming to support MS were done before 2011, and thus had different methodological approaches from what one would expect from more recent psychological research. They thus typically lack preregistrations, statistical power, open data and analysis code, and have a number of measures and conditions that leave much room for researcher degrees of freedom (Wicherts et al., 2016). Some recent attempts at preregistered replications of MS effects have failed (Klein et al., 2019; Pepper et al., 2017; Rodríguez-Ferreiro et al., 2001; Sætrevik \& Sjåstad, 2022; Schindler et al., 2021; Trafimow \& Hughes, 2012). 
Similar to TMT, psychoanalytic theories have also stated that behavior was often motivated by the need to resolve death anxiety. These theories tend to emphasize how people resolve death anxiety through unconscious "psychological defense mechanisms" (Cramer, 1998; Paulhus et al., 1997). Psychological defense mechanisms are described as unconscious mental processes that repress anxiety and other distressing emotions. Defense mechanisms that protect against death anxiety are typically known as primary defense mechanisms, because they develop naturally early in life (American Psychological Association, 2018). If these defense mechanisms are not sufficiently developed, the awareness of being mortal will cause great psychological stress.

Past literature has thus assumed that conscious (and unconscious) awareness of death is so painful that people change their actions, attitudes, and motivations to avoid confronting this thought or suffer great distress.

\section{Earlier work on mapping emotions and attitudes toward death}

Empirical and theoretical research has over the last decades attempted to study and describe people's relation to their mortality by classifying common emotions and attitudes toward death and dying. Spilka et al. (1977) derived eight different commonly occurring beliefs about one's own death through factor analysis of item responses from a Christian sample $(N=328)$. Four of these beliefs were negative in valence (pain and loneliness, forsaking others, failure, a journey to the unknown), one was neutral (indifference), and three were positive (a natural end, an afterlife of reward, courage). Somewhat similarly, Hoelter (1979) developed a "multidimensional fear of death scale" for indexing different types of fear related to death. This scale contained eight dimensions derived from factor analyses of responses from an American undergrad sample $(N=375)$, with items drawn from other scales and his previous work. These dimensions were: Fear of being destroyed, fear of premature death, fear of conscious death, fear of the unknown, fear of the dying process, fear of the dead, fear for significant others, and fear of the body after death. Later, Benton et al. (2007) grouped Hoelter's eight subscales into two distinct types of death anxiety: Existential death anxiety, related to ceasing to exist and what might happen after death, and tangible death anxiety, related to the physical body and the process of dying.

From a psychoanalytical perspective, Langs (2004) postulated three forms of death anxiety based on clinical and theoretical work. These were: Predatory death anxiety due to fear of being harmed or annihilated, which triggers the fight or flight response; predator death anxiety due to fear of harming or annihilating others, which evokes feelings of guilt; and existential death anxiety due to awareness of being mortal. More recently, Petty et al. (2015) created a "death perspective scale" based on work by Kastenbaum and Aisenberg (1976). This scale was designed to measure two opposing perspectives on death: The overcoming perspective, believing that death is the end of one's existence, associated with feelings of defeat and surrender; and the participating perspective, believing that death is a natural process and marks the beginning of an afterlife, associated with feelings of acceptance and honor.

\section{Research needs}

In sum, people's relation to death has gained much interest in both psychology and philosophy. As discussed above, various literature has recurrently argued that the anxiety people experience toward death is quite unique and of great importance to people's mental health, religiosity, cultural attitudes, life decisions, and more. Some (e.g., Becker, 1973) have even argued that resolving death anxiety is one of the core motivations to how people operate and make decisions in their daily lives. However, the empirical foundation for the claim for death anxiety's unique impact remains weak. Few studies have reported on people's actual experiences when confronted with their mortality, and fewer yet while controlling for other negative events. Moreover, although there have been attempts at categorizing and mapping death thoughts, these tend to be directed by a preconceived theoretical framework that limits the range of empirical observations. It would therefore be worthwhile with exploratory analyses comparing responses about death to those about other distressing events. Qualitative and quantitative 
analyses of such responses can provide a better understanding of what may be unique about how people relate to death.

\section{Current study}

The current work presents a dataset of free-text responses where $N=396$ Americans describe their emotions to the thought of dying, and $N=407$ Americans describe their emotions to the thought of having toothache. We aim to explore the contents of these responses, with particular interest in seeing how the respondents describe their experience of thinking about death. Toothache therefore functions as a control condition, allowing us to discover the potentially unique characteristics of people's emotions related to death compared to their emotions related to physically painful experiences.

\section{Hypotheses and preregistration}

The main aim of this work was to provide a qualitative exploration of the text responses to death and to curate the public dataset. We nevertheless wanted to explore whether the emotional content of the responses can be used to address a priori claims about what emotions the thought of death would provoke. Before the quantitative analysis we therefore preregistered two opposing hypotheses (https://osf.io/sw6md). The first hypothesis (H1) stated that "death" would lead to less overt expression of negative affect (operationalized as $\mathrm{H} 1 \mathrm{a}$ for more negative and $\mathrm{H} 1 \mathrm{~b}$ for less positive emotions for "toothache" than for "death"). The second hypothesis (H2) stated that "death" would lead to more expression of negative affect (operationalized as $\mathrm{H} 2 \mathrm{a}$ for more negative and $\mathrm{H} 2 \mathrm{~b}$ for less positive emotions for "death" than for "toothache"). Table 1 presents a $2 \times 2$ layout of the different outcome predictions.

$$
\text { Negative emotions Positive emotions }
$$

\begin{tabular}{lll}
\hline $\begin{array}{l}\text { H1: "Toothache" causes } \\
\text { expression of most negative affect }\end{array}$ & $\begin{array}{l}\text { H1a: More negative } \\
\text { emotions for "toothache" } \\
\text { than for "death" }\end{array}$ & $\begin{array}{l}\text { H1b: More positive emotions } \\
\text { for "death" than for } \\
\text { "toothache" }\end{array}$ \\
\hline $\begin{array}{l}\text { H2: "Death" causes expression of } \\
\text { most negative affect }\end{array}$ & $\begin{array}{l}\text { H2a: More negative } \\
\text { emotions for "death" than } \\
\text { for "toothache" }\end{array}$ & $\begin{array}{l}\text { H2b: More positive emotions } \\
\text { for "toothache" than for } \\
\text { "death" }\end{array}$
\end{tabular}

Table 1: A summary of predictions from the two opposing hypotheses for two different types of outcomes.

\section{Methods}

\section{Research setting}

The data described in the current article were originally collected as free-text responses to the manipulation questions in an experiment on mortality salience (see Sætrevik \& Sjåstad, 2022, for report of the experiment). The original experiment concluded that the manipulation did not produce the expected MS effects. However, the responses to the manipulation questions were not examined in the original experiment and have therefore not been studied in detail. A qualitative analysis of the text responses from the original experiment allows us to explore what emotions the respondents report experiencing when thinking about death. The plan for the current study was preregistered after completing the report of the original study, but before examining the content of the text responses.

\section{Procedure}

Data collection: Eight hundred and three American adult participants were recruited with Amazon's Mechanical Turk (MTurk; Buhrmester et al., 2011; Hauser \& Schwarz, 2016) to answer a Qualtrics 
online survey (Qualtrics, Provo, UT). The sample size and selection criteria were determined by the original study. From the original 803 respondents in the raw data file, we excluded 19 respondents who were marked with identical ID numbers as other respondents. The far majority of the respondents provided relevant and considered responses. However, after the two rounds of coding, 13 responses (eight from the toothache condition) were not considered possible to interpret and classify. ${ }^{1}$ All excluded responses are included in the public dataset, classified as "other" (unclassifiable responses) or marked as "duplicate" (duplicate ID numbers). The final selection consisted of $N=380$ responses for the death condition (237 female, 0 other, mean age 37.7) and $N=391$ responses for the toothache condition (238 female, 1 other, mean age 38.9 years).

Design: This study was conducted as a between-group design where the participants were automatically randomized into one of two conditions. For the current analysis, the between-group conditions ("death" and "toothache") functioned as the predictor variable, whereas the distribution of the emotional categories ("positive", "negative", and "neutral or mixed") functioned as the outcome variable.

The responses were collected through an online questionnaire (Qualtrics, Provo, UT). Because the current questions came first in the questionnaire (directly after an informed consent form) the respondents were not influenced by the steps presented later in the experiment. The respondents received two questions about either toothache or death. The first of these questions prompted the respondents to describe their emotions when thinking about this event: "Briefly describe what feelings the thought of [your own death]/[toothache] arouses in you" The second question prompted the respondents to visualize and describe such an event: "Write down, as specifically as you can, what you think will happen to you [physically as you die and once you are physically dead]/[ the next time you get toothache and its aftermath]". During the initial screening of the first 50 responses, we observed that the responses to the second question rarely mentioned emotion and were thus largely irrelevant for our research aim. Therefore, we preregistered that the responses to the first question would constitute our main outcome variable for the current analysis, and the responses to the second question would be used only when they could help clear up ambiguous responses to the first question. The responses to both questions are available in the accompanying dataset.

The respondents were instructed to give brief answers, and to reply with their first, natural response; please see the online materials for the original "mortality salience" experiment for more details on the experiment design (https://osf.io/s $3 \mathrm{wxd} /$ ). Note that these materials also include other measures in the experiment, and the questions that are analyzed in the current article are on pages 2 and 3 in these materials.

Categorization criteria: The contents of the responses were interpreted and categorized according to whether they expressed "positive", "negative", or "neutral or mixed" emotions ${ }^{2}$. The categorization criteria resulted from discussions between the two authors of this article based on a reading of previous work on classifying death thoughts. The criteria were not directly based on any existing framework, but were designed for distinct, non-overlapping categories to address the hypotheses. The suitability of the

\footnotetext{
${ }^{1}$ Examples are "This is moment death is do notfeel the life. Because what is death and how the feel that for whole life" and "A person who has a severe illness may experience thanatophobia because they are anxious about dying, though ill health is not necessary for a person to experience this anxiety".

2 The "neutral or mixed" emotion category was called "neutral" emotion in the preregistration.
} 
categorization criteria was tested on the first 50 responses before we preregistered the criteria and used them to categorize the full sample. This procedure resulted in the following criteria:

- Responses were classified as "negative" emotion if they explicitly stated experiencing states of negative affect (e.g., fear, sadness, anger), stated the desire to avoid the event in question (death/toothache), or described the event as an undesirable experience.

- Responses were classified as "positive" emotion if they explicitly stated experiencing states of positive affect, stated the desire to experience the event in question (death/toothache), or described the event as a desirable experience.

- Responses were classified as "neutral or mixed" emotion if they explicitly stated experiencing indifference, uncertainty, feelings of conflict, equally positive and negative emotions, or if they responded by avoiding the question.

Meaningf ul responses that avoided answering the question were classified as "neutral or mixed", rather than excluded from the analysis, in order to assess whether they showed indications of psychological defense. Responses that indicated that the respondent had misinterpreted or not understood the question were classified as "other". The content of these responses indicated actual misunderstanding, rather than defensive avoidance. These thirteen responses were excluded from the analysis, but are available in the dataset.

In responses that mentioned both positive and negative emotions, the categorization followed the emotion that was expressed with the most intensity. This was done because we were most interested in capturing the respondents' overall most dominant emotion(s), as these emotions are most significant for our research purpose. The classification of "neutral or mixed" was only used in cases where a response did not clearly suggest that one emotional valence was more dominant or intense than the other. The coding decision was furthermore based on what the respondent described as the truly experienced emotion, not what they tried or wished to feel or think (e.g., "it makes me feel sad, but I try to be brave").

During the first coder's process of categorizing of the full dataset, the following criteria were added to the instructions in order to resolve ambiguities in the predefined criteria:

- Responses were also classified as "negative" or "positive" emotion based on stating to have negative or positive reactions to thinking about death or toothache (e.g., "thinking about death makes me want to cry").

- Responses were also classified as "neutral or mixed" emotion if they expressed emotions that do not have a clear negative or positive valence (e.g., curiosity, acceptance, boredom).

Coding process: All responses were first categorized by one coder (author SS) based on the preregistration (https://osf.io/sw6md) and the adjusted approach, as described above. After examining and discussing these results (authors SS and BS), we preregistered an additional coding of the responses by a second and third coder to increase the validity of the coding. The second coder (AP in the acknowledgments) was kept naïve to the categorization and frequency of each code used by the first coder, as well as to the study's overall theoretical approach and research question. The inter-rater reliability between the first and second coder was high (.901). The majority of the mismatches were between the use of "neutral or mixed" and "negative" codes. The responses with mismatched coding were examined by a third coder (author BS), who determined the final coding of these responses based on the study's categorization criteria and instructions.

We also preregistered a computer-assisted text analysis to assess the validity of the final coding (https://osf.io/zjnd4). The aim was to assess the validity of the human coding by measuring whether the responses that were categorized as positive and negative did in fact contain a higher frequency of 
positive and negative words. The computer analysis used the same dataset as the human coders, analyzing the first question and excluding the responses categorized in the final human coding as "other". We used the "tidytext" package and the "bing" sentiment lexicon for R to perform the analysis. This analy sis counted the number of words in each response that were recognized as positive or negative by the sentiment lexicon (excluding stop-words). We then calculated a ratio for the number of positive words plus total number of words, over the number of negative words plustotal number of words (minus one). The $\mathrm{R}$ analysis script for this operation is available at OSF (https://osf.io/hngck/). The computerassisted analysis supported the validity of the human coding by showing that responses categorized as "negative" by the human coders more frequently used words recognized as negative, and responses categorized as "positive" by the human coders more frequently used words recognized as positive. A one-way ANOVA showed that the sentiment score assigned by the computer-assisted analy sis varied significantly between the human coding categories $(F(2,73.32)=52.53, p<.001)$. However, as can be seen Figure 1, there was nevertheless considerable overlap in the ranges of the sentiment scores that were sorted to each category. This shows that the given sentiment score (e.g., a score around zero) could indicate belonging to either of the human coded categories. Thus the automated text analysis supports the validity of the human coding, but shows that the human coding has a better precision, for example by picking up in subtleties in the language or the context that the words were used in.

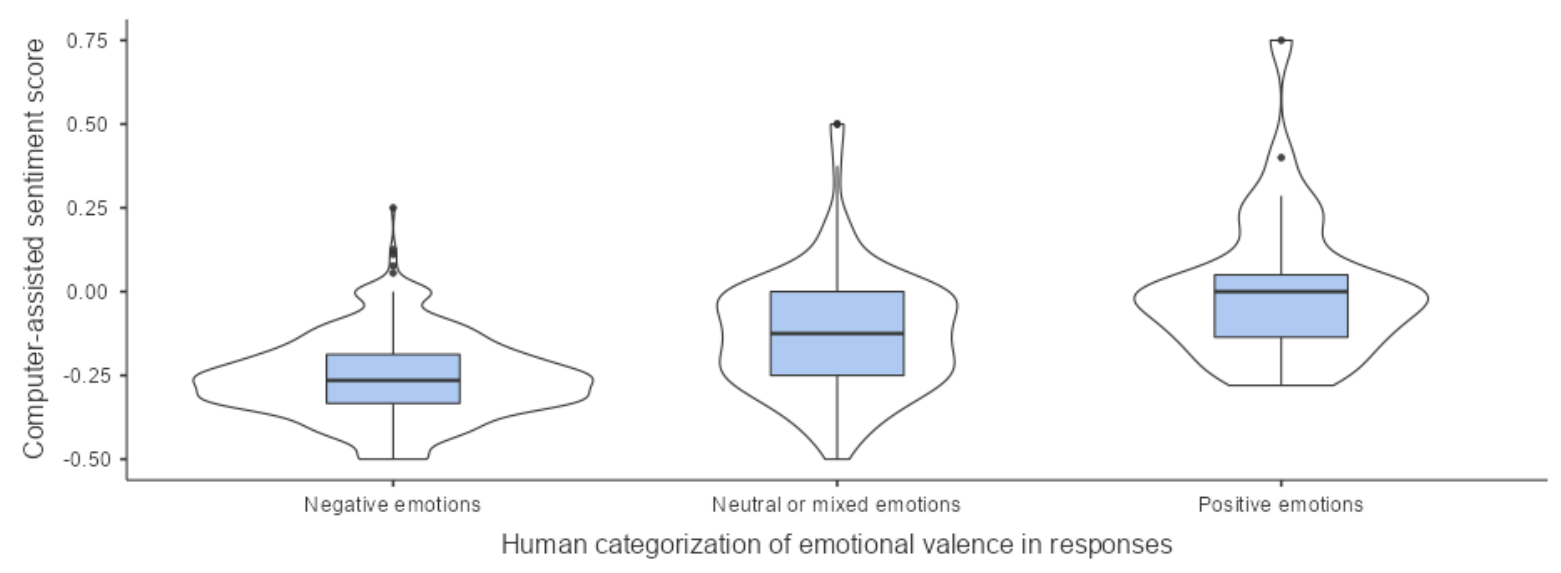

Figure 1: Violin plot comparing human coding to computer-assisted text analysis scores.

The public dataset on OSF (https://osf.io/825gs/) contains the categorizations from all three human coders and their comments about ambiguous responses, as well as the results of the computer-assisted text analysis.

\section{Results}

An initial inspection of the responses indicated that most participants responded earnestly to the questions, and shared personal thoughts. The majority of the respondents in both conditions wrote 2-3 sentences. The responses typically expressed one or more emotions, reactions, or associations to the topic (death or toothache), followed by an attempt to explain their origin or reason. The participants expressed a wide range of sensations, thoughts, and personal experiences related to the topics. Below, we will first provide a preliminary qualitative analysis that reveals more about the response contents in the two conditions. Thereafter, we will provide a simple quantitative analysis that compares the distribution of emotions expressed in the responses.

\section{Qualitative analysis of the responses to toothache and death questions}

Toothache response content: The content of the toothache responses provides us with a basis of responses concerning emotions that are related to an unwanted, but non-lethal event, that we can compare the death responses to. Most of the respondents expressed strong negative emotions to the toothache question. Several respondents even mentioned that they would rather (re-)experience labor 
pains than toothache. Many of the toothache respondents reported that they had experienced severe toothache in the past, although some reported that they had not. Many respondents also expressed a fear of large expenditures related to a dentist consultancy (note that economic concerns may be more pronounced for the MTurk sample than the general population). The specific emotions that were emphasized in the responses varied somewhat, but nearly all of them were negative emotions (see more on the distribution of emotional valence in the section "Distribution of emotions between conditions" below). A typical response in the toothache condition reads: "A toothache arouses several emotions in me. First, annoyance that I am in pain. Then I start to worry about what might be wrong and also whether I should wait it out or go to the dentist. I also get upset and stressed over how much it will cost to fix it."

There were no positive responses to the toothache question, and only few neutral and mixed responses. The neutral and mixed emotion responses usually either avoided answering the question (such as "Wow! I haven 't had a toothache in years. I need to make an appointment with the dentist") or stated that they did not experience any specific emotion (such as "Ifeel no emotions at the mere thought of a toothache. A toothache in actuality would arouse concern "). ${ }^{3}$

Death response content: The death responses expressed a wide range of emotions, thoughts, and mindsets. A great majority of these responses expressed more than one emotion, although most of these emotions were negative. Still, ambivalent (or mixed) emotions, or even positive emotions associated with death was not uncommon in the responses.

In general, we found that many of the respondents mentioned their opinions about what happens after death even when discussing their emotions to the first question. The respondents frequently discussed possibilities such as going to heaven, reincarnation, and nothingness, although most of them also expressed being uncertain about their confidence in these possibilities. When examining the responses, we also discovered two frequent, but opposing, types of statements: Some statedexplicitly that they had no fear of death, while others stated that they were afraid of death. Furthermore, there were a few respondents who distinguished between their personal relation to dying and their concern about the effect their death would have on others. One respondent explained: "Ifeel sad that my loved ones won't have me in their lives. Personally, I am okay with the concept of my own death."

Of the death responses that were classified as negative emotions, the most commonly expressed emotions were fear, anxiety, concem, and sadness. However, there was a wide range of arguments and mindsets behind these emotions. Some recurring themes in the death responses were: (a) Sadness, disappointment, or concern for not yet having achieved their goals in life; (b) fear or anxiety of what might happen when and after they die; (c) sorrow for leaving their loved ones behind; (d) concem or sadness on behalf of those left behind, especially children; (e) unease and confusion due to lacking comprehension and control associated with dying; and (f) fear of ceasing to exist, being "gone forever". Somewhat less frequently, respondents also expressed (g) fear of pain when dying; (h) a feeling of emptiness; (i) a feeling of helplessness; (j) a feeling of disturbance or discomfort; and (k) being reminded of their loneliness. A typical response expressing negative emotion reads: "I feel sadness, confused and anxious. Confusion of what is death and how does it occur. Anxiety of what life after death will be. Sadness of leaving my loved ones and the empty space that I would leave never to be filled again" (expresses the mindsets e, b, and c).

Most of the responses sorted in the "positive" category expressed one of these mindsets: (1) Relief at seeing the end of a life they view as hard or painful (some of these responses mentioned living with

\footnotetext{
${ }^{3}$ Note that this response was coded as "neutral or mixed" rather than negative because this study aims to exa mine the emotions aroused when thinking about a particular event, not when experiencing it.
} 
chronic disease); (m) excitement related to having faith in a positive religious outcome, such as arriving in heaven and meeting God, Jesus or deceased loved ones; or (n) contentment due to feeling ready to die. One of the responses in this category reads: "Honestly, the thought of my own death brings me relief. My life is a roller coaster and it's hard to deal with sometimes. I don 't want to die, but it would be relief from life" (expresses mindset 1).

Responses sorted in the "neutral or mixed" emotion category usually expressed one (or more) of the following mindsets: (o) Conflicting emotions, usually a combination of the negative and positive emotions described in the previous paragraphs; (p) indifference toward death, or simply no distinct emotion; (q) acceptance of death, often expressed as a mindset that death is nothing to fear; (r) apathy or numbness due to having difficulties with grasping the concept of dying. Somewhat less frequently, a few of the responses in the neutral or mixed category also (s) responded rather in a neutral or avoidant manner, without mentioning emotions. One neutral/mixed response in the death category reads: "Current emotions I'm feeling if I were to die todayare no emotions. I don 't feel anything and I wouldn't care if I did die today. I've come to accept that no one will live forever and that death is part of life" (expresses the mindsets $\mathrm{p}$ and $\mathrm{q}$ ).

\section{Distribution of emotions between conditions}

Classifying the responses in both conditions according to emotional valence resulted in 376 "negative", 15 "neutral or mixed", and 0 "positive" responses in the toothache condition versus 238 "negative", 108 "neutral or mixed", and 34 "positive" responses in the death condition. Notably, in the toothache condition, almost all responses were negative, a few were neutral or mixed, and none were positive, whereas in the death condition, the majority of responses were also negative, but there were a substantial number of neutral and mixed emotions as well as some positive emotions. The relative distributions for both conditions are shown in Figure 2.

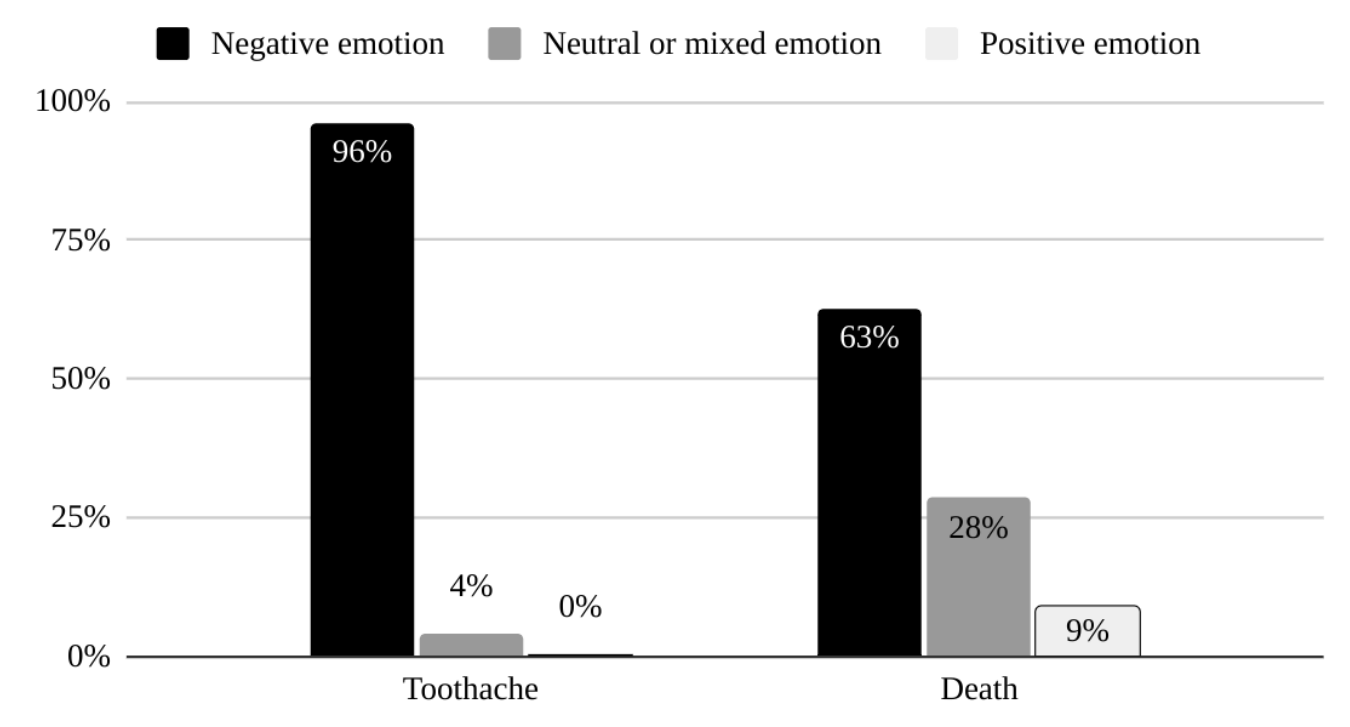

Figure 2: Proportion of the responses in each experiment condition that expressed mostly negative, neutral or mixed, and positive emotion, respectively.

When comparing these distributions, we see a clear difference between the conditions. The observed trend is compatible with hypotheses $\mathrm{H} 1 \mathrm{a}$ and $\mathrm{H} 1 \mathrm{~b}$, showing more negative emotion for toothache than for death, and more positive emotions for death than for toothache. Figure $2 \mathrm{We}$ thus reject $\mathrm{H} 2$ and proceed to test $\mathrm{H} 1$ by testing the significance and size of the difference in positive and negative emotions for between the two kinds of topics. To do this, we performed two separate $\chi^{2}$-tests of association, one comparing the ratios of negative emotion between the groups (testing H1a, negative emotion) and one 
comparing the ratios of positive emotion between the groups (testing $\mathrm{H} 1 \mathrm{~b}$, positive emotion) 4 . The tests were conducted in accordance with our preregistration.

In the first test, we evaluate hypothesis H1a by examining the distribution of "negative" emotions versus non-negative emotions (i.e., either "positive" or "neutral or mixed" emotions) in the two conditions. This $\chi^{2}$-test for negative versus non-negative emotions showed $\chi^{2}(1, N=771)=133.62, p<.001$, indicating that significantly more negative emotions were expressed in the toothache condition than in the death condition. The effect size showed a Cramér's V of .416, which may be considered a moderate effect size (Cohen, 2013).

In the second test, we evaluate hypothesis $\mathrm{H} 1 \mathrm{~b}$ by examining the distribution of positive emotions versus non-positive emotions (i.e., either negative or neutral/mixed emotions) in the two conditions. This $\chi^{2}$-test for positive versus non-positive emotions showed $\chi^{2}(1, N=771)=36.6, p<.001$, indicating that significantly more positive emotions were expressed in the death condition than in the thache condition. The effect size showed a Cramér's V of .218, which may also be considered a moderate effect size (Cohen, 2013).

The two $\chi^{2}$-tests thus showed a statistically significant difference between the two conditions, showing that our respondents reported experiencing both less negative emotion and more positive emotion if they were thinking about death than if they were thinking about toothache.

\section{Discussion}

\section{What characterizes people's relation to death?}

In sum, we observed that people's relation to death is both diverseand affected by a multitude of factors. While the toothache responses expressed quite uniform feelings of distress, there was a much greater variety of sensations and mindsets expressed in the death responses, as observed in both our qualitative and quantitative analyses. Furthermore, our analyses indicated that most people appear to have a considered conception of their mortality; that is, that they are aware of and have devoted some thought to their mortal existence. Their feelings related to this awareness not only varies substantially between people, but also usually involve a wider range and higher number of co-existing emotions than people's feelings towards physical pain. Our results also suggested that experiencing disinterest and apathy is more common toward death than toward pain, but these experiences are far from the dominant attitude toward death in the population.

As for the themes expressed in the death responses, many of them coincided well with dimensions and categories of death thoughts postulated by both Spilka et al. (1977) and Hoelter (1979). For example, mentions of pain and loneliness, an afterlife of reward, fear of premature death, fear of the unknown, fear of the dying process, and indifference all occurred regularly in the death responses. The respondents more often focused on ceasing to exist and leaving things behind rather than on the process of dying. It can thus be said that their mindsets correspond more closely to existential death anxiety rather than tangible death anxiety in the taxonomy of Benton et al. (2007). Comparing our responses to the perspectives on death described by Petty et al. (2015), we observed both responses reflecting the overcoming perspective, those who saw death as the end of one's existence, and responses reflecting the participating perspective, those who saw death as a passage to an afterlife. Compared to the psychoanalytical perspective of Langs (2004), our findings mainly concern what he termed existential death anxiety, ratherthan predator and predatory death anxiety. This could be because the current survey

\footnotetext{
${ }^{4}$ Negative and positive emotion are analyzed separately because the relative ratio of neutral or mixed emotion may vary between the two conditions.
} 
questions specifically asked about emotions related to the awareness of being mortal, and not emotions caused by threats of harming others or being harmed.

\section{Exploring indications of death anxiety}

The results from our quantitative analysis revealed that the respondents who were asked to describe their emotions when thinking about their own death ex pressed considerably more positive emotions and less negative emotions than the respondents who were asked to describe their emotions when thinking about having a toothache. These results supported hypothesis $\mathrm{H} 1$, showing that death led to less overt expression of negative affect than toothache. On the other hand, there was little support for hypothesis $\mathrm{H} 2$, that death lead to more expression of negative affect. We consider this observation intriguing, as it appears to conflict with the common assumption that dying would be worse than experiencing physical pain.

To discuss the theoretical implications of this finding, we will highlight some themes from our qualitative analysis and compare our findings to past assertions about death thoughts and death anxiety. Death anxiety as terror: In existential philosophy and terror management theory, the construct of "death anxiety" indicates that the idea of death is so terrifying to humans that it has a profound effect on most aspects of people's lives, and on culture in general (Becker, 1973; Kierkegaard, 1849; Nietzsche, 1901; Pyszczynski et al., 2015). Different theoretical perspectives on this theory may have made different predictions for exactly how such death anxiety would manifest itself in a study such as this one. However, in our reading of the results, we do not find particular indications of a universal and paralyzing death anxiety, as it is described in most existential theories and the TMT. Below we will discuss few different reasons for this.

Firstly, the fact that because most of our respondents expressed negative emotions to death may initially be taken to indicate that Becker (1973) and TMT (Pyszczynski et al., 2015)'s construct of death anxiety was expressed in our data. However, a crucial element of this construct is the assumption that this form of anxiety is inherently more distressing and terrorizing to humans than other forms of anxiety. However, our results showed that significantly fewer respondents expressed emotional distress if they were asked to think about death than if they were asked to think about toothache.

Further, when we more closely examined the death responses that reflected negative emotion, we found that these responses typically expressed common and moderate emotions, and not "terror"-like experiences to the thought of dying. We observed feelings such as fear, anxiety, and unease, which were typically related to reasonable and valid concerns related to dying (such as leaving loved ones, consequences for others, not reaching life goals, or facing unfamiliar prospects). We rarely observed emotions that were described as unbearable, terrifying, severely distressful, or any thing that would more closely resemble the psychological "terror" described in existential literature. Even more, out of the two conditions, it was the toothache responses that came across as expressing the strongest negative emotions.

Psychological defense mechanisms: One could argue that the respondents in our study could, in fact, have been influenced by terror-like death anxiety, but that these emotions were made unconscious by psychological defense mechanisms which concealed it from the written responses. According to the theoretical models of psychological defense, the se mechanisms work to protect the consciousness from grasping concepts that are too terrifying for people's conscious awareness, such as the fact that they one day will die (Cramer, 1998; Paulhus et al., 1997). Although theories suggesting that one phenomenon is present but concealed by a different phenomenon quickly suffer from falsification challenges, some 
might still claim that psychological defense explains why the death group generally expressed less emotional distress in the responses than the toothache group.

Further inspection of our data nevertheless weakens our belief that psychological defense was present to conceal death terror among the current respondents. Firstly, because the existential theories typically describe death anxiety as a universal fear, we would expect that the psychological defense mechanisms affected most respondents in the sample to numb their suffering, not just the minority who did not express any negative emotions. At least, we would expect the negative emotion responses to be characterized by appearing somewhat less genuine or disassociated with the "self". However, the negative emotion responses in the death group appeared to be both genuinely experienced and personal reactions to the prospect of death, such as fear of ceasing to exist, sorrow for leaving "too soon", or fear of the aftermath of death.

Secondly, we would expect that if defense mechanisms had been prevalent in our sample, we would also have seen more apathy, denial, or having difficulties with responding properly to the questions. Although there were a few cases of this, it was only expressed in a few of the responses, even if we include responses classified as "neutral or mixed". Furthermore, one might also have expected that expressing spiritual beliefs would greatly affect the respondents' emotions to death, considering that resorting to religion is described by many existentialists as a common way to resolve death anxiety (Becker, 1973; Kierkegaard, 1849; Nietzsche, 1901; Pyszczynski et al., 2015). However, even in a sample from a relatively religious population (the U.S.), the majority of those expressing spiritual beliefs were not precluded from also expressing negative emotions.

Hence, we interpret the current results as showing little indication that there was a universal and terrorizing death anxiety present among the respondents when they answered questions about their own death. Neither does our reading of psychological defense mechanisms appear to satisfyingly explain the lack of terror-like experiences we would expect from these responses. Instead, it appears that the reason the death responses reflected less negative emotion and more positive emotion than the toothache responses is that thinking about having toothache is in fact more emotionally distressing thinking about dying.

Possible reasons why death causes less emotional distress than toothache: One cue to why thinking about death induced more positive, neutral, and mixed emotions than thinking about toothache in the experiment may lie in the different nature of the two experimental conditions. While having a toothache is an event that is expected to induce "real" pain, death is instead a form of "nothingness". The philosopher Epicurus argued: "Death (...) is nothing to us, since while we exist, our death is not, and when our deathoccurs, we do not exist" (as cited in Konstan, 2018). Based on this philosophy, Epicurus argued that death is nothing to fear. Following this reasoning, it is reasonable to believe that thinking about death would not always induce negative affect because one would not be there to experience it when it has occurred. Reasoning along these lines appears to be present in some of our participants' responses (e.g., "[I am] not sure about what emotions I feel. I won't know I am dead. A void.”). This mentality could partly explain the different patterns of responses in the two conditions.

Nevertheless, many of the responses reflected that people associate dying with much more than "nothingness". The death respondents of ten reported that their emotional reactions to death were influenced by various dimensions such as their degree of satisfaction with life, past experiences, age, health, family situation, and more. Therefore, death may represent widely different things to people with different life conditions and experiences. For example, some people may perceive death as an abrupt hinder to fulfilling various life goals, while others may have already reached these milestones, and thus feel more prepared to die. Furthermore, some people may regard death as separation from numerous joys in life, while others may perceive their life as more burdensome, and instead associate death with relief and the cessation of pain. 
We also observed that many of the responses that expressed positive, neutral, and mixed emotions to the thought of death referred to religious prospects, such as arriving in heaven, meeting God, meeting passed relatives and friends, and more. It therefore appears that some people might use spiritual beliefs to relieve negative emotions caused by thinking about death, such as suggested by many existentialist theories (e.g., Becker, 1973; Kierkegaard, 1849; Nietzsche, 1901; and Pyszczynski et al., 2015). However, we are skeptical to the claim that not using methods to reveal death anxiety necessarily leads to severe, terror-like distress, beyond the scope of other more casual fears and concerns. Our responses reveal that even the respondents who did not suggest that spiritual beliefs significantly affected their death-related emotions were still able to put their negative emotions into words and describe them in a restrained manner, suggesting that they might not compensate by using other defense mechanisms. We also observed that these people did not have a general tendency to describe particularly terrorizing emotions, but rather emotions that might occur regularly for other reasons in daily life.

In sum, we believe our data indicates that most people have more complex, sophisticated and honest relationships to death than what is suggested by existential theorists. Rather than leading exclusively to aversion of defense, the thought of death can induce a vast range of emotional reactions depending on current life conditions, experiences, and more. We believe that the findings from this dataset are difficult to combine with theidea of a universal and terrorizing death anxiety characterizing people's relationship with death. Instead, we postulate that people's emotions toward death may be far more varied, and are rarely more negative, than their emotions toward a psychical pain.

\section{Implications}

Theoretical implications: The current results fail to support the idea that resolution of death anxiety is common enough to be described as a primary human motivation, and suggest that human behavior and cultural values should rather be explained in alternative manners. If future, more targeted studies also fail to support the idea of the universality of death anxiety and defense mechanisms, the current TMT framework should be modified or abandoned. The need for high-powered tests of TMT's basic assumptions has already been suggested as a consequence of the failed attempts at replicating MS effects (see e.g., Klein et al., 2019, and others cited in introduction). Furthermore, the vast diversity of emotional reactions and attitudes toward death expressed in our sample implies that there may not be universal laws for how people relate to death, and that searching for such laws is a dead end.

The current study has presented hypothetical scenarios of death and toothache, not actual threats. It is reasonable to believe that more vivid, authentic, or visual stimuli would have had different effects on the participants. Therefore, our conclusions may be limited to people's personal perceptions of their reactions to hypothetical death and toothache, and we should be careful in drawing conclusions about how people would react if those situations should become personally relevant for them. Nevertheless, the theories of existential psychology (Becker, 1973; Kierkegaard, 1849; Nietzsche, 1901) tend to argue that the mechanisms are pervasive across people's mundane experiences and influence most aspects of their lives. Moreover, a crucial component of the TMT is that even indirect references to death would motivate the defensive thoughts and behaviors. If so, one would expect even the artificial and hypothetical scenarios used here to evoke at least some of the effects in question.

Proponents of existential psychology may argue that expressions of terror-like death anxiety or defense mechanisms would emerge only in clinical interviews, where the volunteered information must be interpreted and interrogated further by a skilled clinical analyst. Further, it is in the nature of dualprocess theories (Evans \& Stanovich, 2013) that it may be challenging to determine whether a phenomenon (i.e., death anxiety) is absent, or if it is present but negated by a different phenomenon (i.e., defense mechanisms). Unfortunately, such interpretations of the theories make them unfeasible to test empirically in the scale and approach attempted here. Confronted with such criticism, we can merely state that we failed to find any clear positive indication of these phenomena in the current approach. 
Applied implications: This dataset may provide valuable insight into average Americans' mindsets and emotions toward death. It may be particularly useful to reflect on these issuesfor anyone whoencounter such themes as part of their professional life, such as healthcare workers, psychologists, and religious leaders or spiritual advisors. Our data show that it is not uncommon to have mixed, complex, or even positive emotions toward death. It is therefore important for both professional workers, relatives, and others to normalize and legitimize these feelings when speaking to people about such subjects. Further, such interaction may get off on the wrong foot if they approach these issues expecting everyone to have suppressed and hidden feelings about death or that they attempt to reveal some underlying death anxiety.

Methodological implications: The study shows that most people seem able to express their personal emotions when completing surveys online, even on uncomfortable topics. This is not necessarily intuitive, as it has been argued that interpersonal interaction is necessary to study such topics. Despite recent concerns about the quality of MTurk data collection (Chmielewski \& Kucker, 2019; Webb \& Tangney, 2022), the far majority of our sample were willing to provide fairly extensive, coherent and thought-out free-text responses. Although they should be supplemented with other types of study, the use of online surveys may necessary to efficiently provide sufficient statistical power to examine these potential small effects.

We failed to find clear indications of death "terror" or suppressed death terror in our study. Thus, although questions about the participant's relation to their death is a frequently used manipulation in when testing for MS effects, this qualitative analysis indicates that it may not in fact make death thoughts more salient. This may partly explain the failure to support TMT mechanisms in recent experiments (e.g., Klein et al., 2019, and others cited in introduction). Therefore, future research should consider alternative manipulations for activating death thoughts. A minimum requirement should be to perform checks that the manipulation has the direct effect of activating death thoughts, in addition to the indirect effect of influencing other behavior.

\section{Limitations}

Data source: The current study used secondary data that were collected for a different purpose (Johnston, 2017). We believe that this reuse of an extant dataset constitutes efficiency in research, respects the time invested by the participants, and allowed for a larger dataset than we would get for a dedicated data collection for the current research question. However, a novel data collection designed to address our specific research questions on thoughts about death, existentialism, and defense mechanisms could have had other advantages, for instance by including supplementary questions about religious views, the importance of death in their everyday lives, personal experiences with death, and more. The original research design thus limits the scope and depth of the current analysis.

Study design: Altogether, our participants appeared to be able to express personal thoughts and emotions in the responses. However, because the study is based on self-report, the participants could have been affected by social expectations, social desirability, or pressure from culture or religion to express certain attitudes or emotions, especially on the topic of death.

The death and toothache conditions are assumed to be comparable as they both present unwanted events affecting oneself. This experimental design is aligned with how these tasks are typically used in TMT or MS experiments. However, one may be concerned about whether toothache is sufficiently emotionally evocative and vivid to the participants compared to the topic of death. Our examination of the responses indicates that most participants could recollect and reimagine having toothaches. A few respondents reported never having experienced toothache or being more concerned with the financial implications than of the pain involved. Nevertheless, we believe this has only a minor impact on the overall results, as the majority thought of toothache as highly evocative and associated with great pain. 
Generalizability: The sample was recruited through MTurk, which is largely self-recruited, rather than attempting to reach representative samples of the population. The sample may therefore contain some deviation from the average population (Chandler \& Shapiro, 2016). However, studies have shown that MTurk participants are significantly more diverse than the typical college samples often used in similar studies (Buhrmester et al., 2011).

Attitudes toward death may be highly affected by culture, religion, and social norms. Therefore, the variables measured may vary greatly across the world. In this study, the participants consisted of American citizens. The sample is expected to have largely Christian beliefs and values, as were mentioned in some of the responses. As discussed above, religious beliefs may lead to more positive connotations to death, which may increase the proportion of positive and mixed responses in the death condition. It could be that a more secular population would have fewer positive emotions. Moreover, many respondents expressed concerns about how a toothache would affect their economic situation. It is therefore possible that reactions to toothache would vary among different socio-economic settings or countries with different healthcare systems. It is unclear how physical pain and economic concerns would interact in other socio-cultural settings.

\section{Conclusion}

The current findings question some of the basic assumptions of influential philosophical and psychological theories on how people relate to the thought of their own. We found that the respondents self-reported significantly more positive and less negative emotions toward death than toward physical pain. This result gives little support for the idea that death anxiety is a uniquely distressing or unmanageable concern. Furthermore, the phenomenon of psychological defense mechanisms does not appear to satisfyingly explain this underreporting of negative emotions toward death. Instead, our analyses suggest that people's relations to death are diverse, complex, and dependent on many factors, and the corresponding emotions are both conscious and possible to express for most people. Nevertheless, we should note that this article presents only a superficial inspection of the response contents. More stringent and sophisticated qualitative analyses of the dataset may draw more certain conclusions, and the dataset will be available for this purpose. The current dataset give an interesting and unique insight into normal people's relations to their mortality, and we encourage other researchers to further explore the dataset for deeper insights. 


\section{References}

American Psychological Association. (2018). Primitive defense mechanism. In APA Dictionary of Psychology. American Psychological Association. https://dictionary.apa.org/primitive-defensemechanism

Becker, E. (1973). The denial of death. Free Press.

Benton, J. P., Christopher, A. N., \& Walter, M. I. (2007). Death Anxiety as a Function of Aging Anxiety. Death Studies, 31(4), 337-350. https://doi.org/10.1080/07481180601187100

Bonthius, R. H. (1948). Christian paths to self-acceptance. King's Crown Press. https://books.google.no/books?id=_ENWAAAAMAAJ

Buhrmester, M., Kwang, T., \& Gosling, S. D. (2011). Amazon's Mechanical Turk: A New Source of Inexpensive, Yet High-Quality, Data? Perspectives on Psychological Science, 6(1), 3-5. https://doi.org/10.1177/1745691610393980

Burke, B. L., Kosloff, S., \& Landau, M. J. (2013). Death goes to the polls: A meta-analysis of mortality salience effects on political attitudes. Political Psychology, 34(2), 183-200. https://doi.org/10.1111/pops.12005

Burke, B. L., Martens, A., \& Faucher, E. H. (2010). Two decades of terror management theory: A metaanalysis of mortality salience research. Personality and Social Psychology Review, 14(2), 155-195. https://doi.org/10.1177/1088868309352321

Chandler, J., \& Shapiro, D. (2016). Conducting Clinical Research Using Crowdsourced Convenience Samples. Annual Review of Clinical Psychology, 12(1), 53-81. https://doi.org/10.1146/annurevclinpsy-021815-093623

Chmielewski, M., \& Kucker, S. (2019). An MTurk Crisis? Shifts in data quality and the impact on study results. Social Psychological and Personality Science, 11, 464-473.

Cramer, P. (1998). Defensiveness and defense mechanisms. Journal of Personality, 66(6), 879-894. https://doi.org/10.1111/1467-6494.00035

Evans, J. S. B. T., \& Stanovich, K. E. (2013). Dual-Process Theories of Higher Cognition: Advancing the Debate. Perspectives on Psychological Science, 8(3), 223-241. https://doi.org/10.1177/1745691612460685

Freud, S. (1915). Thoughts for the times on war and death (Standard Edition, 14). Hogarth Press.

Greenberg, J., Pyszczynski, T., \& Solomon, S. (1986). The causes and consequences of a need for selfesteem: A terror management theory. In R. F. Baumeister (Ed.), Public Self and Private Self (pp. 189212). Springer. https://doi.org/10.1007/978-1-4613-9564-5_1

Hauser, D. J., \& Schwarz, N. (2016). Attentive Turkers: MTurk participants perform better on online attention checks than do subject pool participants. Behavior Research Methods, 48(1), 400-407. https://doi.org/10.3758/s13428-015-0578-z

Hoelter, J. W. (1979). Multidimensional treatment of fear of death. Journal of Consulting and Clinical Psychology, 47(5), 996-999. https://doi.org/10.1037/0022-006X.47.5.996 
Johnston, M. P. (2017). Secondary Data Analysis: A Method of which the Time Has Come. Qualitative and Quantitative Methods in Libraries, 3(3), Article 3.

Kierkegaard, S. (1849). The sickness unto death [Sygdommen til døden] (A. Hannay, Trans.). Penguin.

Klein, R. A., Ebersole, C. R., Cook, C. L., Vitiello, C., Nosek, B. A., Chartier, C. R., Christopherson, C. D., Clay, S., Collisson, B., Crawford, J., Cromar, R., Vidamuerte, D., Gardiner, G., Gosnell, C., Grahe, J., Hall, C., Joy-Gaba, J., Legg, A. M., Levitan, C., ... Ratliff, K. (2019). Many Labs 4: Failure to Replicate Mortality Salience Effect With and Without Original Author Involvement. PsyArXiv. https://doi.org/10.31234/osf.io/vef2c

Konstan, DavD. id. (2018). Epicurus. In E. N. Zalta (Ed.), The Stanford Encyclopedia of Philosophy (Summer 2018). Metaphysics Research Lab, Stanford University. https://plato.stanford.edu/archives/sum2018/entries/epicurus/

Langs, R. (2004). Death Anxiety and the Emotion-Processing Mind. Psychoanalytic Psychology, 21(1), 31-53. https://doi.org/10.1037/0736-9735.21.1.31

Nietzsche, F. (1901). The will to power (W. Kaufmann, Trans.). Vintage Books.

Nilsson, A., \& Sinclair, S. (2018). Death, ideology, and worldview. https://doi.org/10.31234/osf.io/anh8v

Paulhus, D. L., Fridhandler, B., \& Hayes, S. (1997). Psychological defense: Contemporary theory and research. In Handbook of Personality Psychology (pp. 543-579). Academic Press.

Pepper, G. V., Corby, D. H., Bamber, R., Smith, H., Wong, N., \& Nettle, D. (2017). The influence of mortality and socioeconomic status on risk and delayed rewards: A replication with British participants. PeerJ, 5(e3580). https://doi.org/10.7717/peerj.3580

Petty, E., Hayslip, B., Caballero, D. M., \& Jenkins, S. R. (2015). Development of a Scale to Measure Death Perspectives: Overcoming and Participating. OMEGA - Journal of Death and Dying, 71(2), 146168. https://doi.org/10.1177/0030222815570597

Pyszczynski, T., Solomon, S., \& Greenberg, J. (2015). Chapter one-Thirty years of terror management theory: From genesis to revelation. In J. M. Olson \& M. P. Zanna (Eds.), Advances in Experimental Social Psychology (Vol. 52, pp. 1-70). Academic Press.

Qualtrics (June, 2019). (Provo, UT). Qualtrics. https://www.qualtrics.com/

Rodríguez-Ferreiro, J., Barberia, I., González-Guerra, J., \& Vadillo, M. A. (2001). Are we truly special and unique? A replication of Goldenberg et al. Royal Society Open Science, 6(11), 191114. https://doi.org/10.1098/rsos.191114

Sætrevik, B., \& Sjåstad, H. (2022). Mortality salience effects fail to replicate in traditional and novel measures. Meta-Psychology, 6. https://doi.org/10.15626/MP.2020.2628

Schindler, S., Reinhardt, N., \& Reinhard, M.-A. (2021). Defending one's worldview under mortality salience: Testing the validity of an established idea. Journal of Experimental Social Psychology, 93, 104087. https://doi.org/10.1016/j.jesp.2020.104087 
Spilka, B., Minton, B., Sizemore, D., \& Stout, L. (1977). Death and Personal Faith: A Psychometric Investigation. Journal for the Scientific Study of Religion, 16(2), 169-178. https://doi.org/10.2307/1385748

Trafimow, D., \& Hughes, J. S. (2012). Testing the death thought suppression and rebound hypothesis: Death thought accessibility following mortality salience decreases during a delay. Social Psychological and Personality Science, 3(5), 622-629. https://doi.org/10.1177/1948550611432938

Webb, M. A., \& Tangney, J. P. (2022). Too Good to Be True: Bots and Bad Data From Mechanical Turk. Perspectives on Psychological Science, 17456916221120028. https://doi.org/10.1177/17456916221120027

Wicherts, J. M., Veldkamp, C. L. S., Augusteijn, H. E. M., Bakker, M., van Aert, R. C. M., \& van Assen, M. A. L. M. (2016). Degrees of Freedom in Planning, Running, Analyzing, and Reporting Psychological Studies: A Checklist to Avoid p-Hacking. Frontiers in Psychology, 7. https://www.frontiersin.org/articles/10.3389/fpsyg.2016.01832 
DEATH ANXIETY IN A LARGE ONLINE SAMPLE

Possessed the original data: BS

\section{Contributions}

Conceptualized the study: BS

Planned and preregistered study: BS, SS

Coded the data: SS, AP, BS

Conducted the qualitative analysis: SS

Conducted the quantitative analyses: BS

Drafted the article: SS

Revised the article: BS, SS

Approved the submitted version for publication: BS, SS 


\section{Acknowledgments}

We appreciate the collaboration with Hallgeir Sjåstad in collecting the original data, and with Aleksandra Pawelec who performed the task of second coder as a research assistant. We appreciate the contribution of all the participants in providing their honest and personal thoughts and feelings when answering the online survey. We also sincerely appreciate the valuable feedback and suggestions provided by the reviewers and editor of Collabra: Psychology, which helped us to improve the quality of the manuscript. 


\section{DEATH ANXIETY IN A LARGE ONLINE SAMPLE}

\section{Competing interests}

The authors declare no competing interests relevant for the current study. 


\section{Data accessibility statement}

The full dataset, preregistration, descriptive data, and test of preregistered hypotheses can be found on this article's project page on OSF: https://osf.io/gwszx/ 


\section{DEATH ANXIETY IN A LARGE ONLINE SAMPLE}

\section{Figures and tables}

Table 1. A summary of predictions from the two opposing hypotheses for two different types of outcomes.

Figure 1. Violin plot comparing human coding to computer-assisted text analysis scores.

Figure 2. Proportion of the responses in each experiment condition that expressed mostly negative, neutral or mixed, and positive emotion, respectively. 